Hospital Luis Catvo Mackenna.

Cátedra de Pediatría

Prof. Dr. A. Ariztia.

\title{
PERICARDITIS TUBERCULOSA AGUDA DE TIPO ALERGICO
}

Por los Dres. RAUl MATTE L. y SERGlo ibAÑez Q.

La pericarditis tuberculosa constituye casi la única localización de la tuberculosis del corazón: en sí es una entfermedad extremadamente tara. Sigue en frecuencia al reumatismo, se encuentra en el $5 \%$ de los exámenes post mortem (White). De 85 casos citados por Goldberg, de la clínica de Leipzig, 31 eran consecutivos al reumatismo, 16 a tuberculosis, 16 idiopáticos, 11 tenían una enfermedad valvular, 6 sepsis, 3 empiema, 3 nefritis, etc. En otras estadísticas, la taberculosis se considera la causa en un tercio de todos los casos de pericarditis.

Existe una forma aguda y otra crónica; nos referiremos solamente a la forma aguda. Puede set fibrinasa, serofibrinosa o purulenta.

\section{Etiologia.}

La pericarditis es, por lo general, secundaria a la tuberculosis de alguna otra parte del organismo; pueden existir tres vías: $1^{\circ}$ ) Propagación directa desde $l_{a}$ vecindad (tuberculasis del pulmón, de la pleura $\sigma$ de los ganglios). $2^{\circ}$ ) Difusión por vía linfática a partir de los ganglios, y $\left.3^{\circ}\right)$ Vía hematógena.

En lo que se refiere a la sintomatología y anatomía patológica de la pericarditis en general, no nos referiremos por ser de todos bastante conocida.

Se presentan 4 casos de pericarditis tuberculosa de tipo seroso, que caracterizaron por su similitud: abundante de- 
trame y rápida regresión. Todos los niños eran portadotes de una tuberculosis pulmonar primaria, des de ellos ganglionares y los otros dos ganglio pulmonar. En tres casos la lesión era izquierda y en una derecha. La complicación se produjo muy poco tiempo después de iniciarse la primo infección. En dos casos, al mes después del viraje tubetculinc. En otro, a los tres y en 'el cuarto a los cinco meses.

La iniciación en todos los casos fué aguda, con intensa disnea, taquicardia, angustia, cpresión precordial, fiebre entre 38 a $39 \%$. All examen segmentario se encuentra un gtan aumento del área cardíaca, los tonos están apagados y hay hepatomegalia dolorosa. La radiología demostró gran aumento global de la sillueta cardíaca con borramiento de los latidos.

La producción de liquido. apreciada por el aumento máximo de la sombra cardíaca, varió entre 7 y 17 días.

Los frotes pericardiacos se iniciaron junto con la regresión de los síntomas iniciales y fueron en rápido aumento en 4 ó 5 días, para desapatecer al $10^{\circ}$ dia más o menos. Se cyeron con mayor intensidad y frecuencia en la base del corazón y borde izquierdo del esternón.

La sillueta cardíaca se normalizó en dos casos en uno y medio mes. En un caso en tres meses y en lotro caso lleva cuatro meses y aun persiste ligero aumento de lla silueta.

Los signos físicos cardiaccs (apagamiento de los tonos, aumento área, pulso, respiración, hepatomegalia), fueron más rápido en desaparecer, durando, generalmente, 30 días. El electrocardiograma, en el perído agudo, demostró taquicardia sinusal. Desviación del eje eléctrico. Tiempo de conducción normall y signo de alteración miocárdica.

La presión venosa permaneció en límites notmales; en un caso que lleva tres años de evolución. persiste normal.

La sedimentación no freció variación digna de mención.

En dos de lcs casos se presentó concomitant pleuresía serofibrinosa.

En tres de los cases su comienzo y evclución con rápida mejoria, nos hace pensat en su similitud con la pleuresía serofibrinosa de tipo alérgico. Creemos que la patogenia de estos cascs de pericarditis serosa aguda s:a misma de aquella enfermedad, es decir, alétgica, El caso que lleva tres años de evolución; estando actualment? sano, nos comprobaría esta hipótesís. Los otros dos son de corta observación posterior. para afirmar definitivamente su mejcría total. pero su actual estado de normalidad nos hace pensar en su total mejoría. 
El tratamiento consistió en reposo absoluto en cama. No se practicaron punciones pericardíacas.

O. T. - Obs. 44.II8, -8 años. (Ver cuadro $N^{\prime \prime} 1$ ).

Antecedentes familiares: (-).

Antecedentes persanales: Enuresis. varicela, sarampión y adenitis inguinal en la primera infancia.

Artecedentes de contagio: Tuberculosis desconocida.

Enfeumedad sctuil: Desde diciembre del año 1943 ptesenta decaimiento. febriculas. tos discreta y dorsalgia. Consulta al Centro de Salud, en donde se diagnostica taberculosis pulmonar primaria.

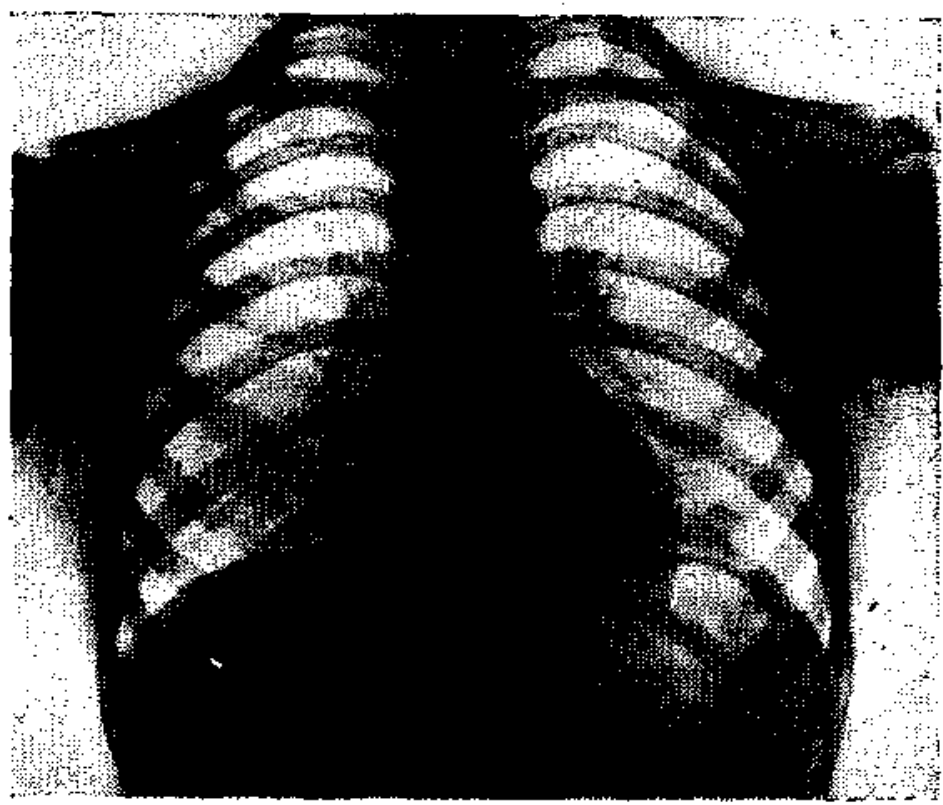

Se hospitaliza el 11 de enero de 1944.

Al examen se encuenta un niño en regulares condiciones, enflaquecido.

Cгілеo y cara: (-).

Fulmón: respitación soplante tercio superior derecho.

Corazón: (-).

Abdomen: (-).

Resto nada especial.

Taberculinas: positivas.

Sedimentación: 18 mn. $x$ hora.

La radiografia demuestra una adenopatía paratraqueal derecha y una infilración infraclavicular de ese mismo lado. 
Evolución: A los primeros días sin molestias, ya a los. 15 días aparecen íebriculas y luego alzas térmicas; el niño está decaído y pálido. At examen fisico solo hay aumento disereto del área catdiaca. La radioscopía tambien revela un aumento global de la silueta cardiaca. Fulso 130 , respicación 30 . La curva febril se mantiene, el área cardiaca aumenta con franco apagamiento"de los tonos." A rayos no' se observan latidos.

A los 17 dias llega la sombra cardíaca al máximo de su aumento $y$ de ese dia se auscultan frotes pericardiacos, la temperatura se normaliza y mejora el estado general. Los frotes pexicardiacos aumentan rápidamente, se acompàñan, a veces. de roces pleutales y desaparecen 15 dias después.

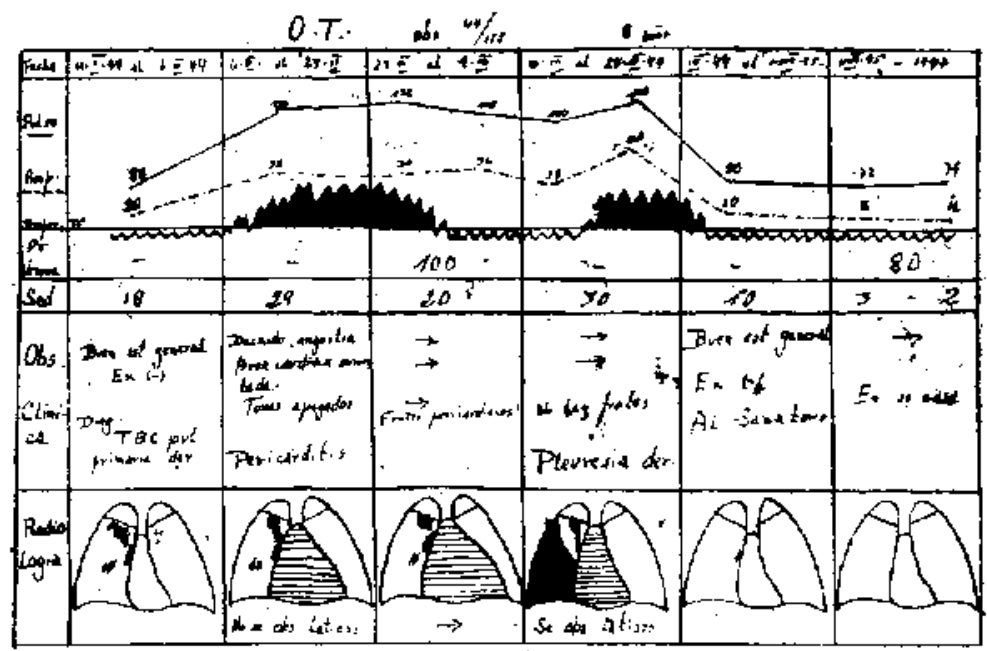

Cuadro $N^{9} 1$.

A los 40 dias de iniciada la perizarditis, la silueta cardíaca recapera su tamañio notmal.

Entre los exámenes practicados durance la enfermedad, el hemograma sóio reveló discretas desviaciones hacia la izquierda. La presión venosa fué de 100 mm.; el electro cardiograma dió: 1) taquicardia sinusal; 2) desviación derecha dil eje eléstrico; 3) tiempo de conducción nonmal; 4) probable alteración mic:cárdica.

Al mas después de este cuadro y estando el niño en buenas condiciones. pero aun sin nomalizarse su silueta cardiaca, presenta una pleuresía serofibrinosa dè regular intensidad y que se teabsorbe en 2 meses.

El 1" de agosto de 1944 , a los 8 meses de hospitalización y con sa lesión utiberculosa en regresión, es enviado al Sanatario San Luis; alti permattece 1 ino y es dado de aita.sano en agosto de 1945. 
Posteriormente, al niño presentó una rifódea, toletándola eq buenas coin. diciones. Actualmente sus controles son (-); no se observa lesión pulme. nat ni cardíaca.

Efecrúa una vida activa, sin ninguna molestia.

La presión venosa es normal.

Electrocardiograma normal (XI1-47).

G. V. - Obs. 255. (Ver cuadro $\mathrm{N}^{2} 2$ ).

Antecedentes familiares: Negativos.

Antecedentes personales: Nacido de técrino. Lactancia normal. Difteria. Coquelucbe en la primera infaucia.

Antecedentes de contagio de tuberculosis desconocido.

Eafermedad actual. - Se inicia en marzo de este año con un eritema tadoso; practicada una radioscopía se observa una adenopatia hiliar izquierda.

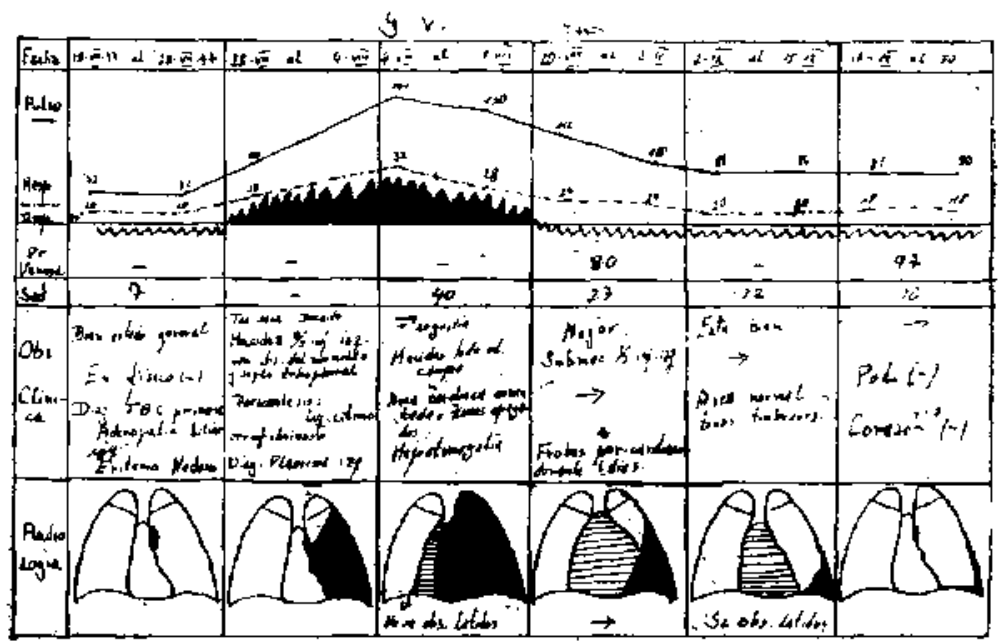

Cuadro $N^{\circ} 2$.

Mantoux $1 \%$ (十+). Ingresa al Sanatorio Josefina Martínez de Ferrari el 16.VII-47, con el diagnóstico de tuberculosis pulmonar primaria. Adenopa. tia hiliar izquierda.

A sa ingreso se encuentra un viño en buenas condiciones generales, con examen físico (-). A las 12 dias se inicia una plectesia serofibrirosa izquierda, comprobada con paracantesis; a la semana después el niño esta muy decaido, angustiado. disneico, taquicartia de 147 . Al examen hay macidez en todo cl campó pulmonar izquierdo y sóplo rubopteural. El área cardiaca se percute a derecha en la linea mamilar y a izquierda se confunde con la macider. pleural. Los tonos cardiacos esrán muy apagados. Higado a 2 cms. 
Visto a radioscopia se encuentra un derrame pleural izquierdo total y in gran aumento global de la sombra cardiaca, sin observarse latidos.

La remperatura disminuye y desaparece a los 20 días, en esa fecha cl derrame regresa bastante, permitiendo observar mejor la sombra cardiaca. El estado general mejora y a los 16 dias de idiciada la perìcarditis se anscultan frotes pericardiacos. Desde entonces nejora bastante, está tranquilo, pulso baja a 100', desaparese la disnea, disminuye lentamente la sombra catdiaca y reaparecen los latidos. Los frotes sólo se anscultan 5 días y desaparecen. La La presión venosa es de $80^{\circ}$.

A los 42 días el pulso. respiración y examen cardiaco es gormal. Sólo persiste una pleuritis marginal izquierda.

En resumen, niño con una tuberculosis primaria. que presenta una plearesia serofibrinosa izquierda, en el curso de 12 ctlal se inotala una pericarditis tuberculosa, que evoluciona a la regresión en 42 dias.

T. M. - Obs. $42 \cdot 3612$.

Se thata de nn niño de 11 años, con antecedentes de contagio tuberculoso, intrafamiliar. Ingresa al hospital el 23-VIY-47. Desde setiembre de 1946 está decaido, anoresia, tos y expectoración. El 10-lil-47, en la Unidad Sanitaria de Quinta Normal. se encuentra una grin adenopatia paratraqueal bilateral de origen tubercalaso. Sedimentakión: 90. Corazón: (一). El 12-VII decae más, febril, se cansa al efectuat ejeccicios $Y$ presenta dolores precordiales. En estus condiciones es traído al hospital e ingresa con el diagnóstiono de pericanditis. Al exanen se encontraba un niño disneico, pálido, decaído. Ban estado nutritivo, elementos de sarna genieralizada. Al examen cardíaco hay gran aumento de su átca, tonos apagados, no se observa latido de la punta. Hígado a 3 cms. Pulso: 140': respiración: 32'. El examen radiolórgico demruestra una adenopatia tamoral izquierda $y$ un aumento de la imagen cardiaca a expensas del pericardio. Presión venosa: 51. Presión arterial: 100 × 80. Sedimentación: 74. Electrocardjograma: taquicardia sinasal. Desviación a izquienda del eje eléctrico. Signos de alteración miocárdica. $T$. de conducción normal.

Los primeros 6 dias de estada continúa ent malas condiciones, sab-febri!, desaido, disnea, angustia. Fulso $y$ respiración muy acelerada. A radioscopia se observa aumento del derrame pericardíaco. A partir del $7^{\circ}$ día se oyen frotes pericardiacos. que vas on aumento tápido $y$ luego disminuyen, para desaparecer en 10 dias. Desde esa fecha mejora tambièn el estado general. eqtá más animado, afebril. El área cardíaca se reduce bastante y los tonos toman su timbre noxmal. Desapatece la bepatomegalia.

La presión venosa ba variado de 51 a 75 a 97 y a 58. tomada cada 20 días mis o menos. Las sedimentaciones se mantienen altas alrededor de $60 \mathrm{~m}$. por hora. 
Actpalmente, 41/2 meses despaes de iniciada so pericarditis, esti en buena- condiciones generales, examen cardiaco (-); pero a radiologia persist: disereto aumento del area. La adenoparia tuberculosa sin variaciones.

R. S. - Obs. 46-4845. - 51/2 años. (Ver cuadro $N^{0} 4$ ).

Antecedentes familiares: Negativo.

Antecedentes de contagio tabercrloso: Positivo.

Antecedentés persoonales: Nacido de parto normal, de término, lactantia notmal, no acasa enfetmedades noteriores.

Enfermedad actual: En noviembre de 1946 se diagnostica toberculoss pulmonar de primo infección. Mabtoux positivo. Ingresa al hospital el $18 \mathrm{~d} z$ diciembre de 1946, se comprueba el diagnóstico, encontrándose sombras difusas er el tercio superior izquierdo y adenopatía hiliar izquierda. Permanece 5 meses y es enviado al Sanatorio Josefina Martinez el 6 de mayo de 1947 .

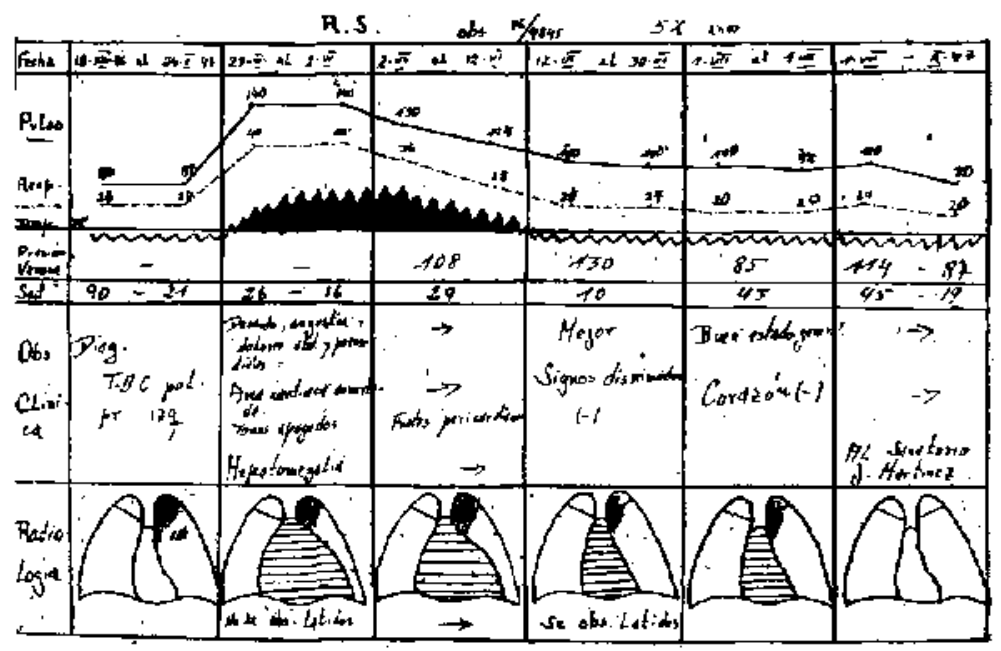

Cuadro $\mathrm{N}^{4} 4$.

En è Sanatorio, se aprecia lesión pulmonar disminuida. Evoluciona sin molestias sólo los. primeros 15 días; después decae, alzas térmicas, vómitos y dolores abdominales. Se agregan' a' estas molestias unj intens palidez, angustias y dolores precordiales. Al examen se encuentra un aumento del área cardíaca, con apagamiento de los tonos, gran hepatomegalia. A tos 10 dias de estar en estas condiciones, el área cartíaca llega al maximo de su aumenco. El niño está muy decaído, con disnea. toquicardia y no se observan latidos al examen radiológico. 'A los ll' dias de enfermedad se auscultan frotes pericar. diacos. Es trasladado nuevamente al Hospital Iuis Calvo Mackenna. 
A su ingreso, el examen no of rece variación, persiste la disnea y taquicardia, gean aumento del área cardiaca, con numerosos frotes pericardíacos. apagamiento de los tonos. Hígado a 4 cms. bajo el reborde costal. Presión venosa: $108 \mathrm{~mm}$. Electrocardiograma: 1) taquicardia sinusal; 2) desviación derecha del cje eléctrico: 3) tiempo de conducción rormal; 4) signos de alteración miocárdica.

A los 18 dias la temperatura be nomaliza, los frotes desaparecen en 10 dias y el niñio mejota su estado general. También el área cardiaca ya disminuyendo y los tonos recuperan su timbre normal.

El estado actual del niño es satisfactorio.

Examen cardiaco: (-).

Lan presiones venosas practicadas cada 20 dias son de 108, 130. 85. 114. 87 .

El electrocardiograma po preøentó variaciones en nn tontrof 2 mesas després.

La alteración que más demotó er normalizarse fué la de hepatomegaitá. que sólo desapareció en 3 meses.

Actualmente está en el Sanatorio Josefina Martínez.

\section{Resumen y conclusiones.}

$1^{9}$ Se presentan cuatro casos de niños portadores de una tuberculosis pulmonar primaria, que se complican de una pericarditis serosa aguda. $2^{\circ}$ Tres de los casos evolucionaron en el curso de uno y medio a tres meses a la completa mejoría. El cuarto caso permanece en el hospital, en observación. $4^{\circ}$ La forma de iniciación. la evolución y rápida mejoria nos hace suponer que se trata de casos de pericarditis tuberculosa de tipo alérgico, simjlar a la pleuresía. $4^{\circ} \mathrm{El}$ diagnóstico de estas pericarditis tuberculosas de tipo alérgico es difí. cil hacerlo en un comienzo. Sólo lia observación protija, la evolución a la completa mejoría ss lo que nos permite precisar la patogenia.

\section{Summary.}

The authors report 4 cases of acute tuberculous pericarditis of serous form in children with primary tuberculosis.

Clinical course was favorable in three cases with complete recovery from one and a hallf month to three months. One case still remains in the hospital.

General symptoms, clinical course and recovery suggest that this cases are similar to tuberculous pleurisy of alergic type. 


\section{Bibliografia.}

P. WHITE. - Heart Disease.

H. ALEXANDER y G. BAER. - Trat. práctico de tuberculasis.

GOLDBERG. - Tuberculosis clínica.

FINE-KATZ. - Dis. of Chest.

RAZ. - Gaz. Méc. de France.

Por un error involustario se omitió en el trabajo intitulado "Tratornos nutritivor crónicos del lactante", publicado en el nuimero anterior, el nombre de los colaboradores del autor. Ellos fueron los Dres, Oscar Correz, Victor Escobedo. Enrique Mujica, Gustavo Ross. Avogadro Aguilera, Raúl Molina. René Alfaro y Raúl Erazo. 